\title{
Acknowledgment of Principal and Ad Hoc Reviewers (2019)
}

\section{Principal Reviewers}

The Editor gratefully acknowledges the following persons who served as Principal reviewers for Personality Disorders: Theory, Research, and Treatment, reviewing a minimum of 3 manuscripts in the past year.

$\begin{array}{llll}\text { Michael Boudreaux } & \text { Matt DeLisi } & \text { John E. Kurtz } & \text { Sarah L. Tragesser } \\ \text { Christopher C. Conway } & \text { Hilary L. DeShong } & \text { Joshua Oltmanns } & \\ \text { Cristina Crego } & \text { Courtland S. Hyatt } & \end{array}$

\section{Ad Hoc Reviewers}

The Editor gratefully acknowledges the following persons who reviewed manuscripts submitted to Personality Disorders: Theory, Research, and Treatment in the past year. Reviewers who reviewed manuscripts under the supervision of a primary reviewer are denoted with an asterisk.

\begin{tabular}{|c|c|c|}
\hline Bravo Adrian & John Edens & Ueli Kramer \\
\hline Jaime Anderson & Julie Eyden & Annegret Krause-Utz \\
\hline \multirow[t]{2}{*}{ Paul Arbisi } & & Sonja Krstic \\
\hline & Sarah Farstad & Janice R. Kuo \\
\hline Bo Bach & Haya Fatimah* & Thomas R. Kwapil \\
\hline Michael Bagby & Elizabeth Flanagan & \\
\hline Arielle Baskin-Sommers & Josh Foster & Jason Lavender \\
\hline Kathy Rae Berenson & & Justin A. Lavner \\
\hline Colleen Berryessa & Kathryn Gardner & Keyne Catherine Law \\
\hline Pavel S. Blagov & Carlo Garofalo & Greg Lengel \\
\hline Robert Bornstein & Sarah Gervais & Richard Linscott \\
\hline Whitney Brown & Jeffrey M. Girard & Benedicte Lowyck \\
\hline \multirow[t]{2}{*}{ Eddie Brummelman } & Claire Gorey $^{*}$ & \\
\hline & Kayla-Ann Gray* & Jacob A. Martin* \\
\hline Elisa Carsten* & & Alexis May \\
\hline Jennifer S. Cheavens & Holly Hamilton & \\
\hline Alexandria Choate* & Jessica Hartmann & Ian $\mathrm{Nel}^{*}$ \\
\hline Anne Conway & Ashley Helle & Craig Neumann \\
\hline \multirow[t]{2}{*}{ David Cooke } & Nathaniel R. Herr & \\
\hline & Perry Hoffman & Thomas Oltmanns \\
\hline Laura Drislane & & Mark Olver \\
\hline \multirow{2}{*}{ Guillaume Durand } & Joshua Jackson & \\
\hline & & Jessica R. Peters \\
\hline Nicholas Eaton & Karen Kelley* & Abigail Powers \\
\hline Ulrich Ebner-Priemer & Nikolaus Kleindienst & \\
\hline David Eddie & Julian Koenig & Lena C. Quilty \\
\hline
\end{tabular}

Babette Renneberg

Juliette Richetin

Julia Richmond*

Camilo Ruggero

Mark Ruiz

Anthony Charles Ruocco

Randall T. Salekin

Shannon Sauer-Zavala

Lori N. Scott

Edward Selby

Andrew E. Skodol

Chelsea Sleep

Antonella Somma*

Joanna Sosnowska

Matthew W. Southward

Tara Spitzen*

Kara Styck*

Matthew Tull

Glenn D. Walters

Mark Waugh 